This item was submitted to Loughborough's Research Repository by the author.

Items in Figshare are protected by copyright, with all rights reserved, unless otherwise indicated.

\title{
The effect of microbiological containment systems on dexterity
}

PLEASE CITE THE PUBLISHED VERSION

PUBLISHER

(C) Taylor \& Francis

LICENCE

CC BY-NC-ND 4.0

\section{REPOSITORY RECORD}

Sawyer, Jo, Allan Bennett, Victoria Haines, Edward Elton, Kathryn Crago, and Sara Speight. 2019. "The Effect of Microbiological Containment Systems on Dexterity”. figshare. https://hdl.handle.net/2134/2845. 
This item was submitted to Loughborough's Institutional Repository by the author and is made available under the following Creative Commons Licence conditions.

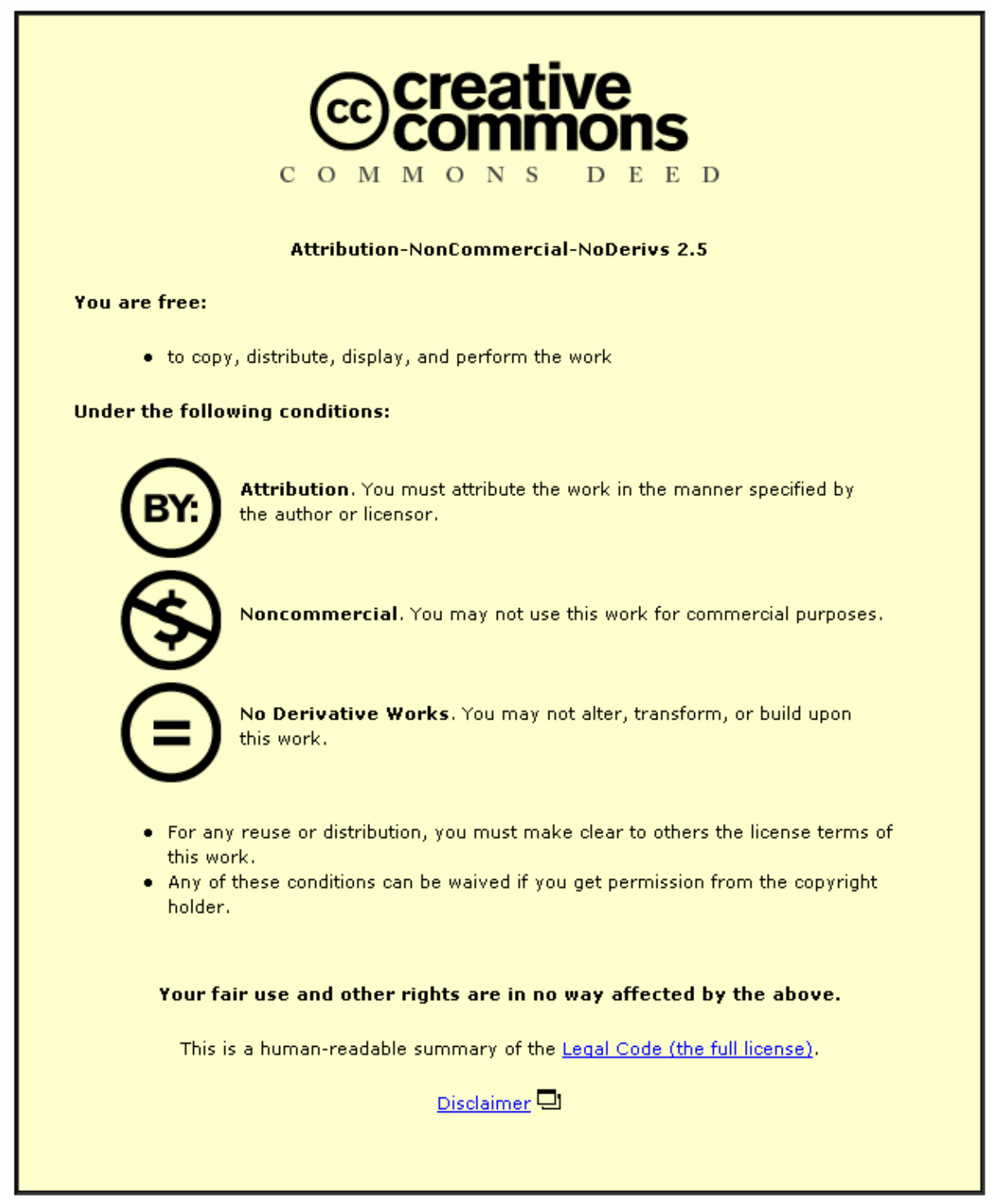

For the full text of this licence, please go to: http://creativecommons.org/licenses/by-nc-nd/2.5/ 


\title{
Measuring the Loss of Dexterity during Microbiological Containment Systems Use
}

Jo Sawyer, Allan Bennett, Victoria Haines, Edward Elton, Kathryn Crago and Sara Speight

Health Protection Agency, Porton Down and

\begin{abstract}
Microbiology laboratories use containment equipment such as safety cabinets and isolators or respiratory protective equipment to protect workers against aerosol infection hazards. There is a perception amongst microbiologists that using sharps within containment equipment would increase the operator's chance of accidental injury due to losses of dexterity within containment and so respiratory protection equipment is the favoured method of containment for these procedures. Using three methods of manual dexterity testing, the effect of latex gloves, a positive pressure respirator, and four forms of containment equipment, a class II safety cabinet, a class III safety cabinet, a half suit and a flexible film isolator were tested against the performance on these tests using bare hands in 20 subjects. With the exception of latex gloves, the personal protective equipment and containment equipment all had a statistically significant detrimental effect on manual dexterity compared to working with bare hands alone. The use of containment systems, especially barrier containment systems such as Class III cabinets and isolators, in the microbiology laboratory significantly reduces dexterity and may increase the chances of accidents occurring within. The use of positive pressure respirators with double gloves also affects dexterity but to a lesser extent. The use of sharps should be minimised within containment equipment. Risk assessment may be required to address the comparative risk of aerosol and needlestick infection with different agents in order to choose the most appropriate containment systems.
\end{abstract}




\section{INTRODUCTION}

The use of pathogenic micro-organisms in microbiology research into the pathogenesis and treatment of infectious disease generates hazards for the laboratory worker. Infection with these agents within the laboratory has been a cause of morbidity and mortality in exposed workers[Collins, 1983 \#31]. Aerosol transmission has long been the most feared route of infection and a range of engineering solutions have been developed in order to control this hazard. These solutions either contain the hazard in an enclosed ventilated area at negative pressure to the operator (primary containment) or provide the worker with personal protection (RPE). The use of negative pressure isolators has been shown to greatly reduce the hazard of aerosol infection in laboratory animal workers[Bennett, $2005 \# 30$ ]. However, there is a perception amongst many equipment users that using sharps (needles, scalpels) within primary containment equipment could increase the operators' chances of accidental selfinoculation.

Sharps injuries are known to be a major cause of blood-borne virus infection in hospitals [Grimmond, 2003 \#19] and the microbiology laboratory[Herwald, 2001 \#33][Sewell, 1995 \#32]. However, due to the limited number of incidents, lack of available records and lack of reporting it is not feasible to measure the number of physical sharps injuries whilst using containment equipment in a controlled experiment. The approach taken in this paper is to measure the comparative dexterity of operators in various forms of protective equipment and in primary containment. Surgical technical performance has been evaluated using dexterity tests [Datta, $2002 \# 14][$ Murdoch, $1994 \# 13]$ and it is proposed that these tests would be a good indicator of laboratory dexterity. Manual dexterity has been measured by conventional tests used in pre-employment screening and occupational therapy. The Purdue pegboard [Tiffin, 1948 \#8] and O'Connor tweezer tests [Hines, 1926 \#9] have been used to quantify the effects of a range of six types of containment strategies on the operator's dexterity, indicating the 
probability of accidents occurring due to loss of dexterity for each piece of equipment.

\section{METHODS}

The effects on operator dexterity of the following six types of containment equipment were assessed.

Table I. Containment equipment assessed and the gloves used with each

\begin{tabular}{ll}
\hline Containment equipment & Gloves used \\
\hline $\begin{array}{l}\text { Latex Gloves - used in standard } \\
\text { microbiology laboratories } \\
\text { Open Fronted Class II Safety Cabinet }\end{array}$ & $\begin{array}{l}\text { Latex laboratory gloves } \\
\text { Double gloves; thicker latex glove } \\
\text { over latex laboratory glove }\end{array}$ \\
Positive Pressure Powered Respirator * Suit Flexible Isolator* - Barrier & $\begin{array}{l}\text { Double gloves; flock lined nitrile } \\
\text { glove over latex laboratory glove }\end{array}$ \\
containment & $\begin{array}{l}\text { Double gloves; flock lined nitrile } \\
\text { glove over latex laboratory glove }\end{array}$ \\
Flexible Film Isolator* - Barrier & Double gloves; arm length heavy duty \\
Class III Safety Cabinet* - Barrier & glove over latex laboratory glove \\
containment &
\end{tabular}

* used in high containment laboratories

The original laboratory dexterity study carried out at HPA Porton Down assessed the effects on operator dexterity of the first five containment strategies listed in table I, compared to working with bare hands alone. This study was then extended to look at the effects of posture in a class III cabinet using the following test conditions:

Bare hands sitting,

Bare hands standing, 
Class III cabinet sitting and

Class III cabinet standing.

\section{Subjects}

Participants for both studies were drawn from HPA Porton Down staff. The subjects had a range of experience of working with containment equipment and $57 \%$ of the subjects tested were scientists by occupation. Anthropometric data and other variables were also recorded in order to identify trends associated with differing human dimensions. Table II illustrates some of the personal data recorded for each part of the study.

Table II. Profile of subjects for both dexterity experiments

\begin{tabular}{lcc}
\hline & $\begin{array}{c}\text { Study of a range of } \\
\text { containment equipment }\end{array}$ & $\begin{array}{c}\text { Study of effects of posture } \\
\text { working in a class III cabinet }\end{array}$ \\
\hline $\mathrm{N}=$ & 10 & 20 \\
Age range & $22-41$ & $22-52$ \\
Gender & 6 females, 4 males & 10 females, 10 males \\
Dominant hand & 9 right, 1 left & 18 right, 2 left \\
\hline
\end{tabular}

In the second study subjects were additionally asked a series of questions to assess the effects of a range of variables including gender, age, tiredness and stress.

\section{Manual Dexterity Tests Used}

Dexterity tests should be selected and results interpreted with caution, because dexterity is not a unitary ability[Fleishman, 1954 \#21]. It has been suggested that dexterity consists of five factors (finger dexterity, manual dexterity, wrist-finger speed, aiming and positioning) and that these factors do not necessarily correlate within an individual. Consequently a battery of tests would give a more accurate reflection of dexterity as affected by containment strategy. The tests chosen in this study provide data on three types of dexterity. 


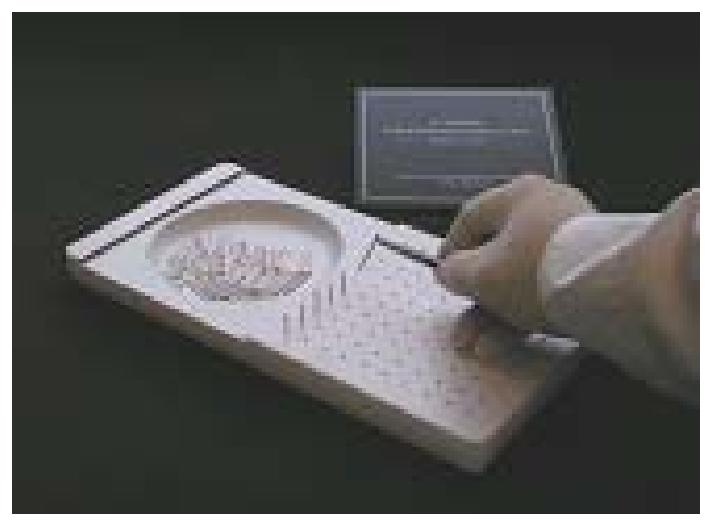

The O'Connor tweezer dexterity test (Lafayette Instrument model 32022) measures the ability to measure skilful arm and hand movements, also described as "manual dexterity"[Fleishman, 1954 \#21]. High scores also indicate precision and steadiness in the use of small hand tools[Hines, $1926 \# 9$ ].

Test administration:

Administration was based on the method described by Hines[Hines, 1926 \#9]. Subjects were asked to place pegs in the holes using tweezers. A score was derived from the number of pegs placed in the board in 5 minutes. To standardize the test, each subject was shown the easiest way to hold and use the tweezers and allowed to have a short practice, in line with test administration procedures.

Purdue pegboard test:

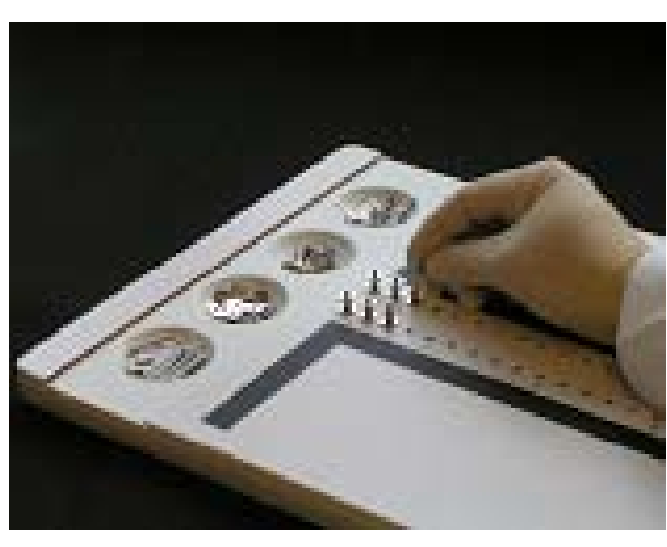

The Purdue pegboard test (Lafayette Instrument model 32020) measures both "gross dexterity" which is the gross movements of hands, fingers and arms, and "fine finger dexterity", defined as the ability to coordinate finger movements in performing fine manipulations[Fleishman, 1954 \#21][Tiffin, 1948 \#8]. 
Test administration:

This was based on the method described previously by Tiffin[Tiffin, 1948 \#8]. Gross dexterity was measured using the combination test, which was recorded as the sum of the number of pegs placed first with the dominant, then the nondominant hand then both hands in the holes in 30 seconds for each part of the test. The assembly test measured fine finger dexterity and required the construction of "assemblies" of washers, pegs and collars using both hands working simultaneously in 60 seconds. To standardize the test, each subject was given short practice before the test began, in line with test administration procedures.

To standardise the tests for height, the bench the subjects worked on was raised for taller people when standing. This meant that taller subjects were not stooping whilst standing to complete some of the tests.

To evaluate any effects of learning or fatigue the first half and second half of the O'Connor Tweezer test scores were recorded and compared, in each containment condition. The administration times of the Purdue pegboard tests were too short to accurately assess learning and fatigue effects in this way.

\section{Statistical Analysis}

Minitab Release 13.32 software (Minitab Ltd) was used for all statistical analyses. Where appropriate, one- or two-way, repeated measures or mixed ANOVAs were performed. Friedman tests were used where parametric assumptions were not met. Dunnett's and Tukey's posthoc tests were used. T tests or Wilcoxon tests were used to compare the means of data and linear regression assessed any correlation between anthropometric measures, questionnaire data and the dexterity test scores. The alpha level was set at 0.05 for statistical significance. 


\section{RESULTS}

\section{Testing the effects of a range of containment strategies on dexterity}

Manual dexterity scores were not significantly different between the first and second half of the test in any of the containment strategies used, suggesting learning and fatigue had no effect and the period of familiarisation given was sufficient.

The range of containment strategies tested significantly reduced manual $(F=15.05, p>0.0005)$, gross $(F=111.12, p=0.0005)$ and fine finger dexterity test scores $(F=58.03, p=0.0005)$. Assessing the effects of the containment strategies in more detail, posthoc testing using Dunnett's test found manual dexterity was only significantly affected by the flexible film isolator $(T=-6.889, p<0.00005)$. Gross dexterity was affected by both the flexible film $(T=-16.65, p<0.00005)$ and the half-suit isolator $(T=-13.55, p<0.00005)$. Lastly fine finger dexterity was significantly reduced by both the flexible film $(T=-11.8, p<0.00005)$ and the halfsuit isolator $(T=-10.77, p<0.00005)$ and the respirator $(T=-9.12, p<0.00005)$.

For each containment strategy, the percentage reduction in each type of dexterity from bare hands was calculated in order to make a valid comparison of the three dexterity types. The interaction between the type of containment and the type of dexterity was significant $(S=32.10, p=0.015$, adjusted for ties). Fine finger dexterity was reduced most of all by containment, followed by gross dexterity and manual dexterity was least affected (figure I). 


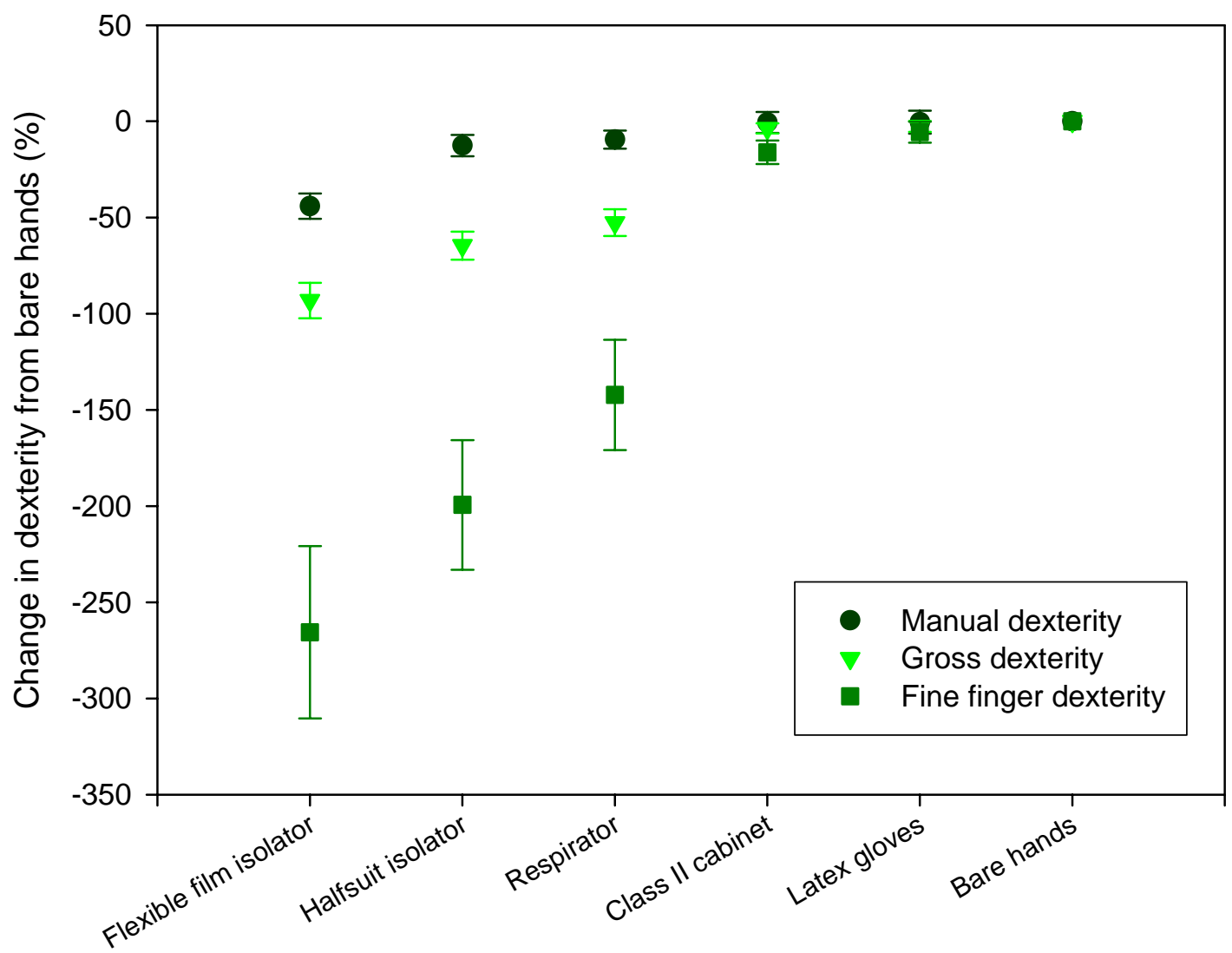

Containment strategy

Figure I. Percentage change in manual, gross and fine finger dexterity with different containment strategies.

\section{Increased time for task completion}

In category III training at HPA Porton Down, workers are taught to work more slowly in containment to reduce the chance of injury or contamination. As shown in figure I working using a respirator on a task requiring gross dexterity would hypothetically increase the time needed to complete the task by $53 \%$. Working flexible film isolator on a task which requires fine finger dexterity would increase the time needed by $266 \%$. Thus for a fine finger dexterity based task taking 10 minutes, in a flexible film isolator it would take nearly 27 minutes to give a comparable level of performance. 


\section{Effect of posture working at a Class III cabinet on manual dexterity}

There was no statistically significant correlation between the anthropometric measurements (limb and hand and finger size, weight, height and BMI) taken and the scores achieved in any of the dexterity tests when testing the effects of posture and working in a class III cabinet on dexterity, under any of the environment conditions used. Additionally no correlation could be found between the test scores achieved and tiredness, stress, age, experience, alcohol, medication or smoking in either part of this study.

Between the four conditions tested, sitting or standing working in a class III cabinet or at a bench, a significant difference was found for manual dexterity $(F=27.2, p<0.0005)$, gross dexterity $(F=174.64, p<0.0005)$ and fine finger dexterity $(S=47.66, p<0.0005$, adjusted for ties).

Tukey's posthoc test found a difference between manual dexterity scores when working on the bench compared to in the class III cabinet $(p<0.00005)$ but no difference between sitting or standing. There was also a difference in gross dexterity scores on the bench compared to in the cabinet $(p<0.00005)$ but no difference between sitting and standing.

The fine finger dexterity data did not meet parametric assumptions, thus a Friedman test was used. However, Wilcoxon t tests showed a significant difference between working sitting at the bench and working sitting in a cabinet (ci=36.5, 43 and 12,16 respectively) and also a significant difference between working standing at the bench and working standing in a cabinet (ci=36, 43.5 and 12,16 respectively). There was no difference found between sitting and standing (figure II). 


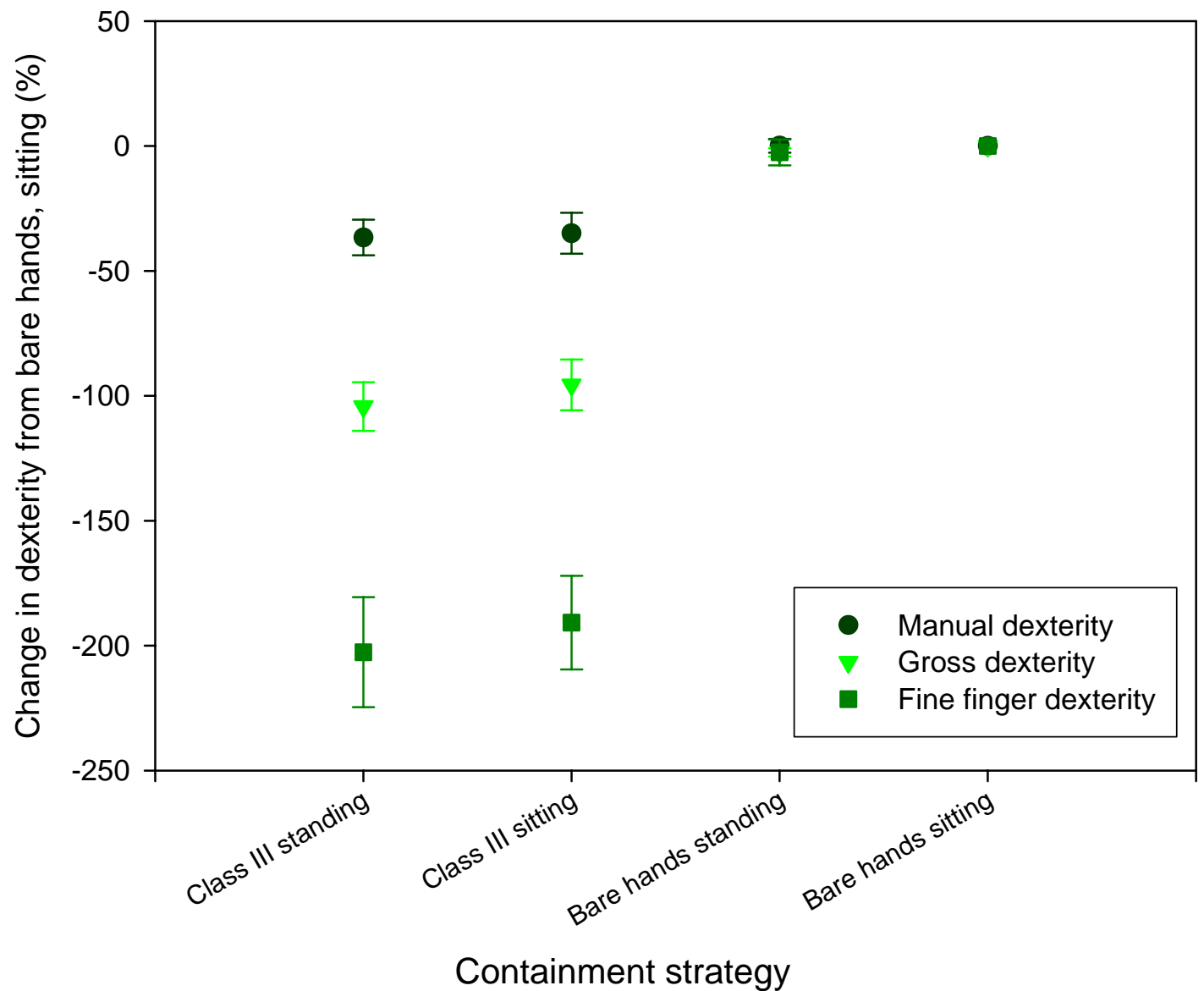

Figure II. Percentage change in manual, gross and fine finger dexterity between working with a class III cabinet and different postures.

The interaction of the three types of dexterity test scores between the four conditions assessed was statistically significant $(S=8.2, p=0.042)$. Thus, reflecting what was found with the range of containment equipment tested, the switch to working in a class III cabinet affects manual dexterity the least, then gross dexterity and had the most detrimental effect on fine finger dexterity. 
Male / Female Differences in Performance on Manual Dexterity

\section{Testing the effects of a range of containment equipment on manual dexterity. Differences between the genders.}

Based on t test calculations there was no statistically significant difference in the measured manual, gross or fine finger dexterity between the genders when using the latex gloves, safety cabinet, respirator with double gloves, flexible film isolator or half suit isolator. Additionally using analysis of variance no interaction could be found between gender and the range of containment equipment used for manual, gross or fine finger dexterity.

\section{Effect of posture working at a Class III cabinet on dexterity. Differences between the genders.}

When using the class III cabinet there was no significant difference in dexterity between the gender groups. A two-way mixed ANOVA also found no interaction between the gender groups and test condition, defined as both posture and the dexterity test completed, when working with bare hands.

However, a graph of the effect of posture on dexterity test scores between the genders (figure III) shows that the mean dexterity test scores for women exceeded the men's scores in every test condition for bare hands.

Using paired $t$ tests and adjusting the alpha level to 0.0083 to account for the six $t$ tests for each test condition, one significant difference was found. For the bare hands test condition women's fine finger dexterity was better than men's when sitting $(\mathrm{t}=3.06, \mathrm{p}=0.008)$. 


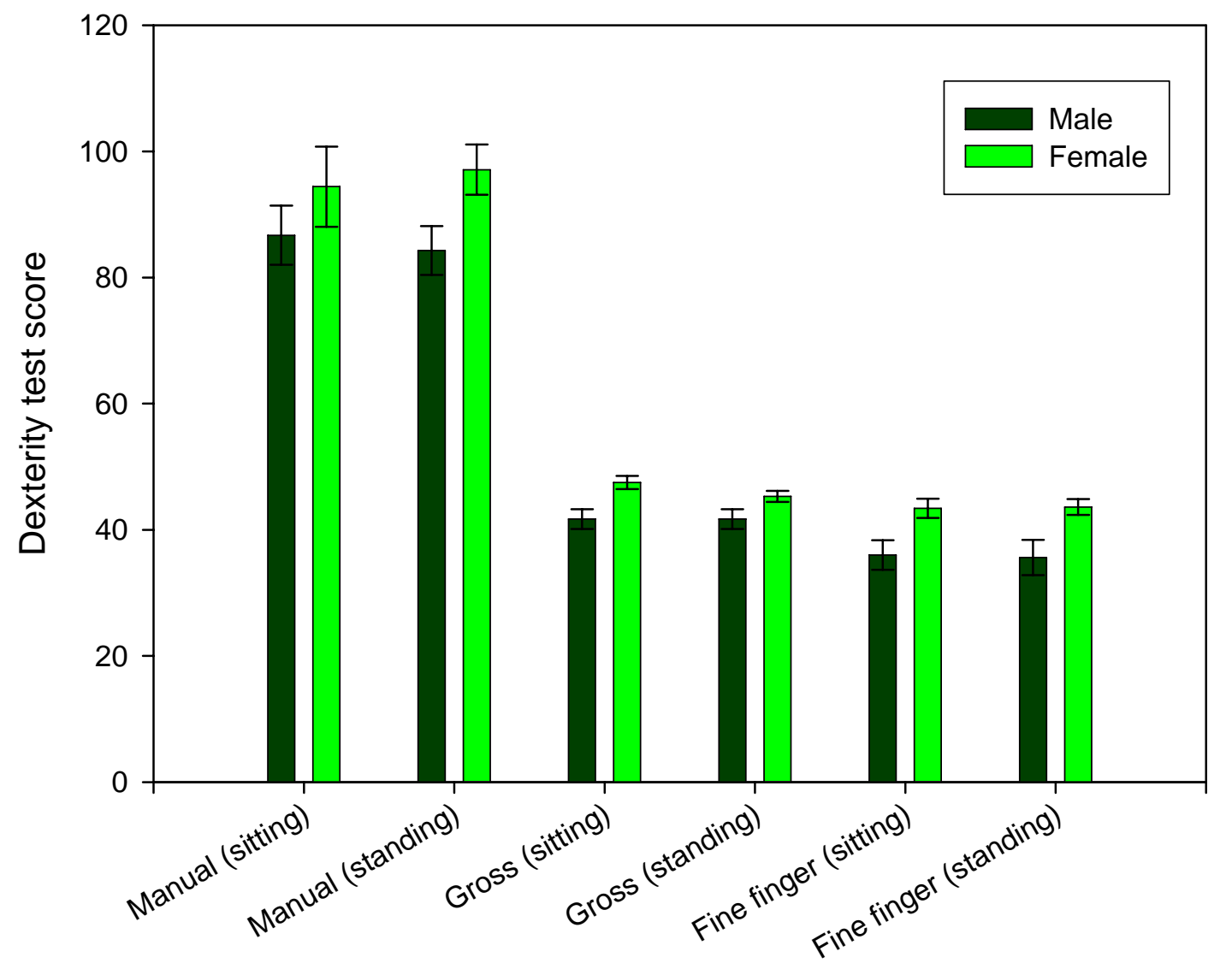

Dexterity type and posture

Figure III. The effect of posture (from sitting to standing) on dexterity test scores between the genders, in the bare hands condition.

\section{DISCUSSION}

The use of standard dexterity tests has provided a means to quantify the comparative dexterity performance of containment measures. These tests have shown that the use of containment strategies can cause significant losses in dexterity of operators. In particular primary containment equipment which have a barrier between the operator and the task (flexible film and half suit isolators, class III cabinets) produce the highest loss of dexterity. This loss of dexterity 
could lead to accidents if sharps are used. An assessment of the comparative risk of sharps accident to that of aerosol infection should be made for any procedures undertaken within containment.

The flexible film isolator was found to reduce manual, gross and fine finger dexterity. The half suit isolator reduced gross dexterity, and the half suit isolator and respirator reduced fine finger dexterity. Laboratory tasks will require different types of dexterity for the operator to work safely, thus the appropriate containment equipment must be selected for the task in hand. Where possible the minimum primary containment may be required in order to have the least impact on tasks requiring dexterity, and therefore safety, particularly where a task requires fine finger dexterity.

The range of containment equipment tested had a more detrimental effect on the gross and particularly fine finger dexterity scores than on the manual dexterity test scores. The thickness of the double gloves and the awkward positioning of the subject as they completed the tests would make small movements of the fingers and picking up and manipulating small objects, as required by fine finger dexterity, more difficult than larger movements with larger objects. Vision is obscured by the containment equipment (glass panels, Perspex canopies and respirator) this would also affect the ability to see small objects more than larger objects.

The use of latex gloves had no effect on the levels of manual dexterity, gross dexterity or fine finger dexterity in this study. In some cases gloves can improve grip, depending on the type of glove, and the manipulation required of the object held[Riley, $1985 \# 12]$. The properties of latex give the glove surface extra friction which may even improve grip and dexterity.

Although there appeared to be no significant learning or fatigue effect during the O'Connor tweezer test based on the data collected, it is probable that these 
effects exist, but are cancelling each other out. In the laboratory situation, a learning effect will not improve performance indefinitely, but worker fatigue has the potential to constantly reduce performance. Some subjects complained of aching arms and hands during the tests when using the containment equipment. Consequently workers should have full training in the use of containment equipment to reduce the chance of unsafe practice during initial use, and workers should take frequent breaks to avoid fatigue.

One study found no link between the dexterity of 34 hospital personnel and the incidence of multiple needlestick injuries[Casanova, 1993 \#29]. However the authors discuss the possibility that some employees with multiple needlestick injuries and impaired hand function may have declined to participate for fear that the data obtained might adversely affect their employment status. Containment equipment which severely reduces an individual's dexterity nevertheless has the potential to increase the incidence of sharps injuries compared to working with bare hands, particularly if the operators are not given a period for training and adjustment. Operators need to learn to work more slowly to compensate for any lack of dexterity when using containment. Indeed operators of containment equipment must be aware they may need to work nearly 3 times as slowly to maintain their level of fine finger dexterity in the highest levels of containment.

No correlation was found between anthropometric measurements or other factors recorded and the dexterity test scores. The other factors recorded were selfreported tiredness and stress, hours of shift worked, smoking, units of alcohol consumed, gender, handedness and current state of health. Extreme tiredness and alcohol would be expected to cause a drastic loss of dexterity. Only one subject had a self reported tiredness score of 8 out of 10 (10 being the most tired), the remainder of the group were below this. The lack of extremes of the factors recorded may explain the lack of any correlation with dexterity in this study. However, extreme tiredness and stress are not usual in the work 
environment and workers in containment should be trained to avoid carrying out dangerous tasks under these conditions. It is also difficult to standardise selfreported factors. There was no relationship between age and dexterity levels either, even though older age groups have been shown to be slower in similar dexterity studies for up to 70 year olds[Ruff, 1993 \#6] and 85 year olds in one study using the Purdue pegboard test[Pennathur, 2003 \#11]. But the upper age range was only 52 for this experiment and the older subjects in this study may have more laboratory experience, which could have improved their scores, contrary to the fact that increased age is associated with reduced dexterity levels.

Additionally, there may also be a complex interaction between the factors recorded and other factors not yet identified that would impact dexterity performance. For example, it was suggested that focus of attention is an important determinant of performance for manual dexterity tests of the nondominant hand[Strenge, 2002 \#2]. Unfortunately the sample size in this test does not allow the analysis of these factors whilst controlling for other factors such as gender, dominant hand or glove size.

There was no effect of posture (defined in this study as sitting compared to standing) on working in class III cabinet conditions or in the bare hands condition. Working in a class III cabinet compared to bare hands not surprisingly reduced manual dexterity, gross dexterity, and fine finger dexterity. Furthermore, class III cabinets also had a greater adverse effect on gross and fine finger dexterity than on manual dexterity. This is possibly because gross and fine finger dexterity were measured using a class III cabinet with glove apertures high up in the front of the cabinet. This made it more awkward to complete the tests as they were placed low down in the bottom of the cabinet. The manual dexterity test however would have been easier to complete at this angle because tweezers are used so the subject's hands can be higher up than for the pegboard tests. Class III cabinets vary in their design and the glove apertures can be in different places. Regardless of whether an operator is sitting or standing, the position of the glove 
apertures and the location of the task within the cabinet must be considered when selecting a cabinet to work in.

For the range of containment equipment tested in the first study there was no gender difference in dexterity levels, although the sample size was small. In the larger, second study there was also no gender difference in dexterity using class III cabinets. However for the bare hands condition, women's fine finger dexterity was better than men's whilst sitting. The difference found between the mean scores of the two genders was always in the same direction, with the females always performing better than the males. Although there was no difference in performance between the genders when using the class III cabinet, women in the study had significantly smaller hands (based on glove size $\mathrm{F}=14.4, \mathrm{p}=0.001$ ) than men and a smaller upper limb length $(F=21.48, p<0.0005)$. This left the women at a greater disadvantage when working in a class III cabinet because the gauntlets tended to get in the way and slip down if the gauntlets were too big for an operator. The gauntlets on the cabinet used were size 9 which explains why the gender difference seen in the bare hands condition disappeared when working in the cabinet. The sizes of gauntlets on class III cabinets at HPA, Porton Down were always selected based on the user with the largest hands. Modifications such as a rubber band to hold gauntlets in place for users with smaller hands, or a cabinet design that allowed gauntlets to be changed between users would improve dexterity in the class III cabinet.

Men have a superior visuo-spatial ability[Vecchi, 1998 \#25], and they perform better at finger tapping tests implying they have faster muscle speed[Ruff, 1993 \#6]. In contrast, women were found to be better at tasks that require a repeated sequence of hand and arm movements made with minimal external sensory guidance (controlled by a left-hemisphere based motor programming system that is called the manual praxis system). It appeared that men rely much more heavily on visual and tactile cues than women in making repetitive hand and arm movements during a task[Chipman, 2002 \#27]. 
In some containment equipment such as the half suit isolator and the class III cabinet, visual and tactile cues are impaired. Conversely, some repetitive tasks undertaken in containment equipment during routine laboratory work may require a degree of manual praxis. This highlights the need for the design and selection of containment equipment with the highest degree of visual and tactile function, to give the optimum dexterity available to both genders.

\section{CONCLUSION}

Primary containment equipment especially those providing barrier containment such as flexible film isolators, half suit isolators and class III cabinets have been shown to affect manual, gross and fine finger dexterity. The use of positive pressure and double gloving also affects fine finger dexterity but to lesser extent than barrier containment. Latex gloves and posture have no effect on the dexterity types measured. Containment equipment should be designed and selected to both fit the task and the operator. Particularly if the task requires fine finger dexterity, a minimal containment strategy should be used. Work should be organised to allow rest breaks or changes of task to reduce fatigue. Sufficient time should be given to the task in hand, with appropriate training and adequate practice.

\section{REFERENCES}

